\title{
Raloxifene for older women: a review of the literature
}

\author{
Helga Hansdóttir \\ Department of Geriatrics, Landspitali \\ University Hospital, Landakoti, \\ Reykjavik, Iceland
}

\begin{abstract}
Raloxifene is a non-steroidal selective estrogen-receptor modulator (SERM) which is used for prevention and treatment of postmenopausal osteoporosis. Raloxifene decreases the incidence of vertebral fractures by $30 \%-50 \%$ in postmenopausal women with osteoporosis but has not been shown to decrease the incidence of hip fractures or other non-vertebral fractures. At the present time, estrogen-replacement therapy and bisphosphonate treatment are the only medical treatments that are proven to prevent hip fractures with the exception of vitamin D and calcium replacement, which has been shown to prevent hip fractures in elderly individuals and nursing home residents. Raloxifene has been shown to have additive effects on bone turnover and bone mineral density (BMD) when used along with alendronate and teriparatide. Raloxifene could have a role in renal failure as it has been shown to increase BMD of the vertebra over 1 year of therapy. Raloxifene is as effective as tamoxifen in reducing the risk of invasive breast cancer. The increased incidence of venous thromboembolism is the main concern of raloxifene therapy and previous history of venous thromboembolism is a contraindication for use of raloxifene. Raloxifene has a role in treatment of vertebral osteoporosis in older women. The decision to use raloxifene should be based on evaluation of fracture risk and on potential other benefits than fracture reduction along with consideration of side effects.
\end{abstract}

Keywords: SERM, raloxifene, osteoporosis, women, fractures, old age

\section{Introduction}

Raloxifene is a nonsteroidal selective estrogen-receptor modulator (SERM) which has been marketed for use in prevention and treatment of postmenopausal osteoporosis. One of the consequences of the Women's Health Initiative has been increased interest in the SERMs, because of the potential to retain most of the beneficial effects of estrogen while avoiding some of the adverse effects (Lawrence Riggs and Hartmann 2003). Raloxifene binds to estrogen-receptors, with estrogen agonistic effects in some tissues and estrogen antagonistic effects in others. In the last few years a number of clinical studies have been published on the effects of raloxifene on osteoporosis, the risk of invasive breast cancer and cardiovascular diseases. There are a number of other SERMS currently under investigation but raloxifene is the only SERM currently on the market for osteoporotic fractures (Vogelvang et al 2006). Below is a review of the effects of raloxifene in some target tissues, results of clinical trials, along discussion of the usefulness of raloxifene in the treatment of osteoporosis in older women.

\section{Mechanisms of action}

There are three interactive mechanism that explain the pharmacology of SERMs: Differential estrogen-receptor expression in a given target tissue, differential estrogenreceptor conformation on ligand binding and differential expression and binding to the estrogen-receptor of coregulator proteins (Lawrence Riggs and Hartmann 2003). There are two main types of estrogen receptors $\alpha$ and $\beta$. The $\alpha$-receptor is mainly an 
activator and $\beta$-receptor inhibitor. The SERMs bind to both receptors, functioning as pure antagonist when binding to $\beta$-receptors and as a partial agonist when binding to the $\alpha$-receptor. The different action of the individual SERM depends on coregulatory proteins and their ability to recruit coactivators. Estrogenic activation inhibits osteoclasts and thereby reduces the bone resorption by osteoclasts that restores the balance between bone formation and bone resorption (Sarkar et al 2002).

\section{Effects of raloxifene on bone}

Raloxifene decreases the markers of bone turnover by $30 \%-40 \%$ after 1 year and increases bone density at several scanning sites by $2 \%-3 \%$ after 3 years in postmenopausal women with osteoporosis (Delmas et al 1997). Raloxifene decreases the incidence of vertebral fractures by $30 \%-50 \%$ (Figure 1) but does not decrease the incidence of hip fractures or other nonvertebral fractures (Ettinger et al 1999). By contrast, estrogen reduces vertebral and hip fractures rate by $34 \%$ over average of 5.2 years of therapy (Anderson et al 2002). Studies that have looked at bone mineral density (BMD), bone architecture and bone turnover suggest that estrogen is more effective antiresorptive agent than raloxifene (Prestwood et al 2000). Raloxifene increases BMD at the hip but studies have not shown decrease in any non-vertebral fracture. Bisphosphonate therapy decreases vertebral fractures by $48 \%$ (Liberman et al 1995) only slightly more than raloxifene therapy does and decreases hip fracture incidence by $30 \%-50 \%$ in postmenopausal women with osteoporosis (Black et al 1996; McLung et al 2001). Bisphosphonates increase bone mineral density (4\%-9\%) considerably more than raloxifene or estrogen-replacement therapy (2\%-3\%) does (Black et al 1996). This difference in bone mineral density increases is not reflected in decrease in vertebral fractures It has been postulated that this discrepancy in effects of bone mineral density and vertebral fracture prevention is due to the normalization of high bone turnover in cancellous bone by raloxifene and prevention of microarchitectural disruption which leads to reduction in vertebral fracture rates. It has been suggested that only low therapeutic threshold is needed to prevent osteoclasts from perforating the trabecular plates and therefore reduce fractures of cancellous bone. Stronger antiresorptive agents would be needed for effects on cortical bone such as the
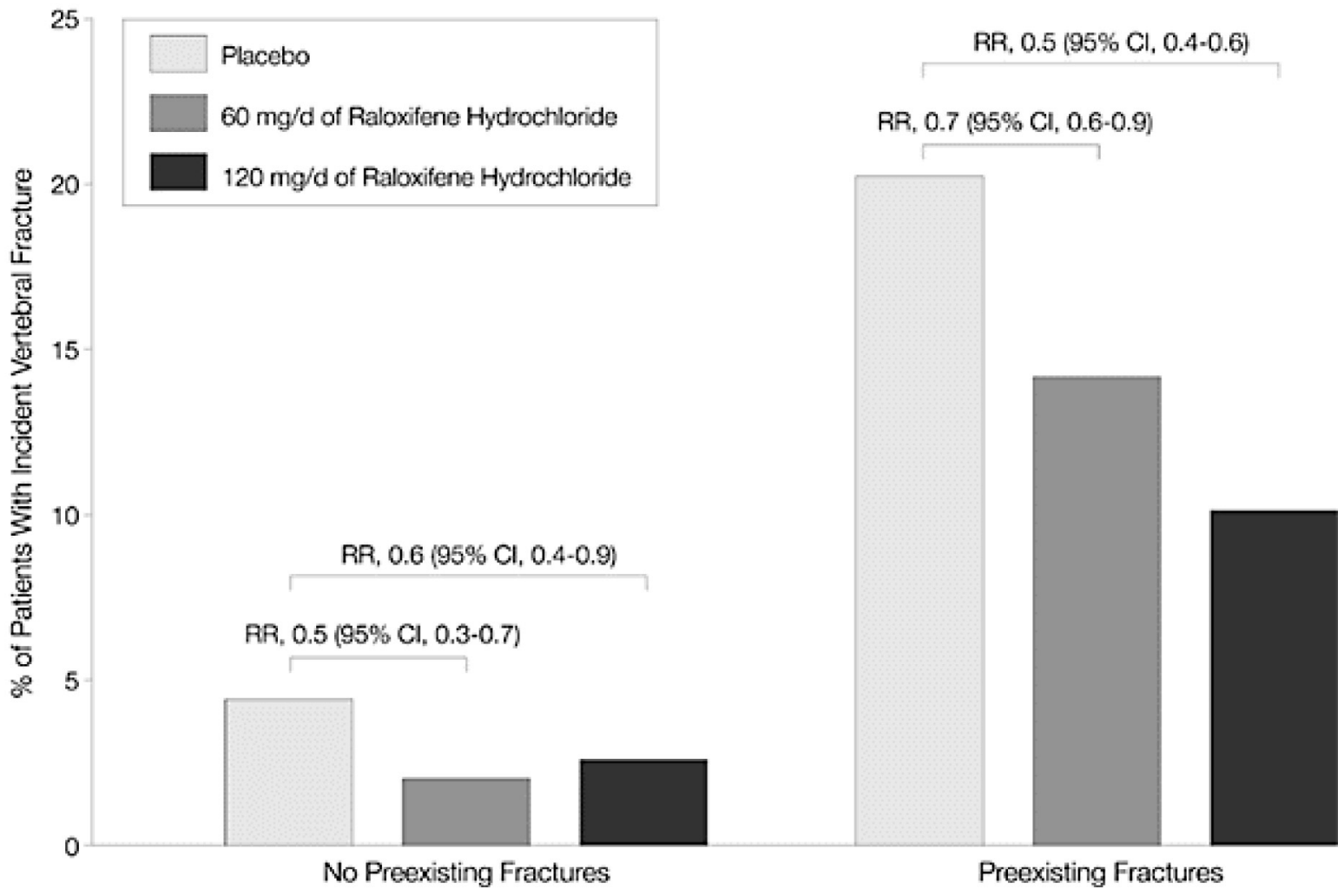

Figure I Reduction in new vertebral fractures among 6828 women who completed the study. Reproduced with permission from Barrett-Connor E, Grady D, Sashegyi A, et al 2002. Raloxifene and cardiovascular events in osteoporotic postmenopausal women. Four year results from the MORE (Multiple Outcomes of Raloxifene Evaluation) randomized trial. JAMA, 284:847-57. Copyright (C) 2002 American Medical Association. 
hip (Sarkar et al 2002). At the present time, adequate vitamin D and calcium supplements (Riggs and Melton 2002), estrogen-replacement therapy (Anderson et al 2004), and bisphosphonate treatment (Black et al 1996; McLung et al 2001) are the only medical treatments that are proven to prevent hip fractures. However, studies on the effect of raloxifene in women older than 70 who are at the greatest risk of hip fractures are not availabe. Raloxifene has been shown to have additive effects on bone mineral turnover and BMD when used with alendronate (Bischoff-Ferrari et al 2005) and teriparatide (Johnell et al 2002) but these studies have not lasted long enough to show fracture reduction.

\section{Effects on breast cancer}

Raloxifene has anti-estrogenic effects on breast tissue, as tamoxifen does. The Multiple Outcomes of Raloxifene Evaluation (MORE) trial, where 7705 postmenopausal women with osteoporosis were randomized to raloxifene or placebo, demonstrated a $76 \%$ reduction of invasive breast cancer over 4 years (Cummings et al 1999). In the Raloxifene use for the Heart (RUTH) trial where 10,101 postmenopausal women with either coronary heart disease or multiple risks for coronary heart disease were randomized to raloxifene or placebo, there was a $44 \%$ relative reduction in invasive breast cancer over median of 5.6 years (Barrett-Connor 2006). Tamoxifen has demonstrated efficacy for treatment and prevention of estrogen-receptor positive breast cancer. The National Surgical Adjuvant Breast and Bowel Project Study of Tamoxifen and Raloxifene (NSAP STAR P-2) trial compared the effect of tamoxifen versus raloxifene over 5 years on invasive breast cancer incidence in 19,747 women at increased risk of breast cancer (Villareal et al 2001). Raloxifene was as effective as tamoxifen in reducing the risk of invasive breast cancer. Raloxifene had lower incidence of thromboembolic events, and cataracts and similar effects on other cancers, fractures, ischemic heart disease, and stroke.

\section{Effects on the genitourinary tract}

Raloxifene does not stimulate the growth of the endometrium nor the vaginal mucosa (Kannus et al 2000). Therefore raloxifene would not be useful for either treating postmenopausal estrogen deficiency symptoms of the lower urinary tract or vagina-like dyspareunia, frequency of urination, urinary tract infections, or urinary incontinence. Tamoxifen as estrogen has been related to endometrial cancer (2.5-fold increase). The STAR P-2 trial demonstrated increased incidence of endometrial cancer in the tamoxifen group compared with raloxifene but without statistical significance (Vogel et al 2006).

\section{Effects on cardiovascular risk factors}

Raloxifene favorably alters biochemical markers of cardiovascular risk by decreasing LDL-cholesterol, fibrinogen, and lipoprotein (a), and by increasing HDL2-cholesterol without raising triglycerides. In contrast to hormone replacement therapy, raloxifene has no effect on HDL-cholesterol and PAI-1 and a lesser effect on HDL2-cholesterol and lipoprotein (a) (Walsh et al 1998). Do these favorable biochemical effects mean protection against cardiovascular disease? In a secondary analysis of the MORE trial, with women with increased risk of cardiovascular risk, cardiovascular events were significantly reduced (Barrett-Connor 2002). These results were not confirmed in the RUTH trial where 10,101 postmenopausal women at risk for cardiovascular diseases were randomized to raloxifene versus placebo (Barrett-Connor 2002). Raloxifene was neither associated with increased nor decreased cardiovascular event. Considering death rates in the RUTH trial, there was a slight significant increase of fatal strokes (but not stroke overall) in the raloxifene group which was offset by a significant decrease in non-cancer and non-cardiovascular deaths in the raloxifene group (Barrett-Connor 2002). While these results are not necessarily true it is wise to consider high risk of stroke a relative contraindication to raloxifene therapy. On the other hand, the presence of ischemic heart disease is neither an indication nor a contraindication for raloxifene therapy.

\section{Age consideration}

The studies on raloxifene include women who are postmenopausal at a mean age of 65-67 (SD 5-7 years) despite women up to 80 years of age being enrolled (Delmas et al 1997; Barrett-Connor 2006). Observation time ranged from 3 years to 6 years, meaning most of the women are middle aged or young-old. Therefore the results apply mainly to middle aged or young-old women rather than old and frail women who are the usual clients of geriatricians. In these studies, however, there are women with cardiovascular disease or risk factors for cardiovascular disease, osteoporosis or increased risk of breast cancer. Such increased risk of poor outcome characterizes the health problems of older people in general, making the results somewhat more applicable to older women. There are a few studies that have looked at older, frailer women and women in nursing homes. Only vitamin D supplements and hip protectors have been shown to be effective to prevent hip fractures in nursing homes (Kannus et al 2000; Bischoff-Ferrari et al 2005). A study on the effect of raloxifene on markers of bone turnover in 
women living in a long term care facility (mean age 84) demonstrated comparable efficacy in decreasing bone turnover as in younger women (Hansdóttir et al 2004). Alendronate (Greenspan et al 2002) and estrogen replacement therapy (Stevenson et al 2005) increase BMD of both hip and vertebra and decrease bone turnover in women living in long-term care facilities and frail elderly women. Risedronate has been shown to be effective in women up to the age of 80 but was not effective in older women who were assigned to treatment based on falls risk but not because of osteoporosis (McClung et al 2001). That does not mean it is not useful in women older than 80 , it means therapy of osteoporosis should be for those who have osteoporosis, falls risk is another entity that needs to be evaluated and treated according to the relevant literature. It is likely that these treatments have identical effects on bone in the oldest and frailest age group but other aspects need to be taken into consideration when deciding how to prevent fractures such as falls risk, mobility, nutrition, comorbidity, and altered drug metabolism.

\section{The use of raloxifene in older women Osteoporosis}

Prevention and treatment of vertebral osteoporosis is the main indication for raloxifene use. Osteoporosis is common in old age and osteoporotic fractures reach epidemic proportion in the highest age groups (Figure 2) especially in nursing homes. Incidence of $10 \%$ fracture rate in one year in a nursing home has been reported (Chandler et al 2000). Osteoporosis should be taken seriously and treated aggressively just as any other health problem. Fractures, both vertebral and and non-vertebral have a large impact on quality of life (Sarkar et al 2002). Oral vitamin D supplementations between 700-800 IU a day reduce the risk of hip and non-vertebral fractures in ambulatory and institutionalized elderly person (Bischoff-Ferrari 2005)). Vitamin D deficiency is common in winter in northern latitudes, especially in those who cannot go outside and needs to be replaced (Steingrimsdóttir et al 2005). Every older individual should be advised upon adequate vitamin D and calcium intake. Raloxifene is a cost effective preventive treatment of osteoporosis in women 50-80 years old irrespective of severity of osteoporosis (Stevenson et al 2005). The cost of a quality-adjusted life year (QALY) is estimated at $£ 20,000-31,000$, which is considered reasonable. These effects assume no effect on hip fractures and are largely driven by the reduction of breast cancer. In the older age groups where hip fractures are more prevalent, bisphosphonates (alendronate and risedronate) are more cost effective treatment than raloxifene with QALY of less than $£ 20,000$ in women at high risk of a fracture but the cost is higher in younger women or in those without previous fractures. The most important reason women cannot take bisphosphonates is because of compliance, gastrointestinal symptoms and occasionally musculoskeletal pain and renal impairment (Emkey et al 2006). In my practice there are a number of women who need nurse's assistance to take medication and once a week special visit for a weekly bisphosphonate would add considerably to the cost of therapy. The availability of a monthly dose is now an attractive choice (Reid et al 2002). Parenteral therapy is possible for those who cannot tolerate bisphosphonates by mouth (Ersoy 2006). Raloxifene is an alternative for vertebral fracture prevention and is easy to administer. It is given once a day and can be given with other

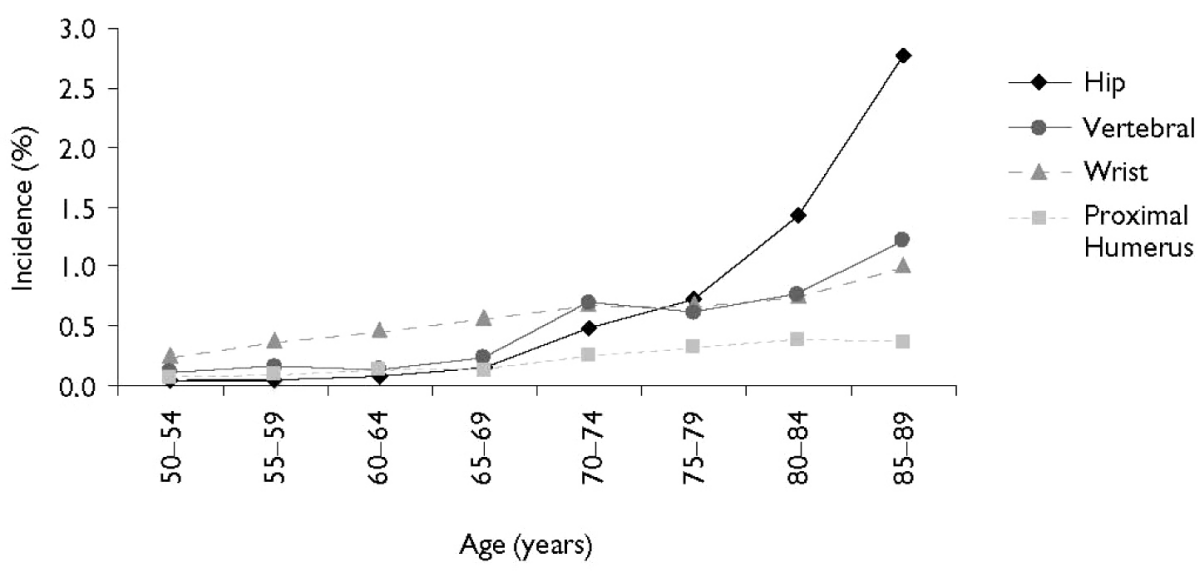

Figure 2 Annual incidence of osteoporotic fracture in females by site. Reproduced with permission from Stevenson M, Lloyd M, De Nigris E, et al. 2005. A systematic review and economic evaluation of alendronate, etidroneate, risedronate, raloxifene and teriparatide of the prevention and treatment of postmenopausal osteoporosis. Health Technology Assessment, 9:1-160. Copyright (C) 2005 NIHR. 
regular medications and has the added benefit of reduction of hormone postive breast cancer. Raloxifene has been used along with other drugs for osteoporosis, such as alendronate (18) and teriparatide (Deal et al 2005). In both cases, raloxifene has an additive effect on BMD in both hip and vertebra along with increased reduction of bone turnover. Renal failure complicates the treatment of osteoporosis (Ersoy 2006). Bisphosphonates and teriparatide are in general not recommended in renal failure if creatinine clearance is less than $30-35 \mathrm{~mL} / \mathrm{min}$. Raloxifene might have a role in renal failure as it has been shown to increase BMD of the vertebra after one year of therapy (Hernandez et al 2003). It is metabolized by liver and is safe to administer in renal failure. The presence of nephrotic syndrome should, however, be looked upon as a contraindication to raloxifene due to increased risk of venous thrombosis. More studies are needed for fracture prevention in renal failure for any agent.

Strontium ranelate and teriparatide are other alternativeson the market for osteoporosis and fracture prevention which have shown reduction of fractures (Neer et al 2001; Reginster et al 2005).

\section{Reduction of risk of breast cancer}

When choosing an agent for treatment of any health problems, other effects have to be taken into consideration. The secondary benefit of raloxifene is prevention of hormone positive breast cancer. There are no current guidelines on primary prevention of breast cancer so raloxifene for that use is not at present indicated for that use. The yearly overall risk of newly diagnosed breast cancer is one in 500 for a 70-year-old woman and 1 in 400 for an 80-year-old woman, that would be approximately 1 in 40-50 over 10 years and 1 in 20-25 over 20 years (McPherson et al 2000). The fact that while breast cancer comprises $20 \%$ of mortality for younger women, other disease such as cardiovascular diseases can be attributed to a majority of deaths for older women and breast cancer only to a small percentage (Cummings et al 1993). The issue of treatment of invasive breast cancer should be referred to a specialist in most cases and therefore beyond the scope of this article. Women with a breast tumor who do not desire neither a work up or anti-cancer treatment such as surgery, radiation nor chemotherapy, could be offered raloxifene with the added benefit of vertebral fracture prevention. Tamoxifen is effective in reducing recurrences of hormone positve breast cancer as a sole treatment (Cummings et al 1993) and raloxifene can be expected to do the same. Such situations arise in nursing homes or in case of very frail old women.

\section{Increase in risk of thromboembolic events}

The increased incidence of venous thromboembolism is the main concern of raloxifene therapy and previous history of venous thromboembolism is a contraindication for use of raloxifene. Old age is risk factors for thromboembolic events reaching approximately $0.5 \%$ a year at the age of 80 (Ageno et al 2006). Raloxifene treatment increases the risk 1.5-3.0 times which would mean absolute risk of $0.75 \%-1.5 \%$ a year (Ettinger et al 1999; Riggs 2000). Over 5 years that could mean 3\%-7\% risk and over 10 years of therapy up to possibly $15 \%$ likelihood of a thrombosis. Other risk factors are obesity, tendency for thrombosis, cancers, inflammatory diseases, and surgery. Hospital stay with immobility, stroke, heart failure, respiratory failure, sepsis or inflammation, increase the risk considerably. It is wise to use active thromboprophylaxis and stop raloxifene treatment during the hospital stay until the patient is fully mobile for these conditions.

\section{Conclusion}

The decisions to treat osteoporosis for fracture prevention needs to be discussed with the individual and the fracture risk needs to be taken into account along with other benefits and risks of treatment. Every older individual should be advised upon adequate calcium and vitamin D replacement. Individuals at increased risk of fracture either because of osteopenia or osteoporosis should be evaluated for further treatment. Raloxifene is an effective treatment for prevention of vertebral fractures in postmenopausal women. Raloxifene has the added benefit of prevention hormone positive breast cancer but increases risk of venous thromboembolism. Raloxifene could be used as well as an additive agent in postmenopausal women with severe osteoporosis.

\section{Disclosures}

The author has no conflicts of interest to disclose.

\section{References}

Ageno W, Squizzato A, Garcia D, et al. 2006. Epidemiology and risk factors of venous thromboembolism. Sem Thromb Hemos, 32:651-8.

Anderson GL, Limacher A, Assaf AR, et al. 2004. Effects of conjugated equine estrogen in postmenopausal women with hysterectomy: the Women's Health Initiative randomized controlled trial. JAMA, 291:1701-12.

Barrett-Connor E, Grady D, Sashegyi A, et al. 2002. Raloxifene and cardiovascular events in osteoporotic postmenopausal women. Four year results from the MORE (Multiple Outcomes of Raloxifene Evaluation) randomized trial. JAMA, 284:847-57.

Barrett-Connor E, Mosca L, Collins P, et al. 2006. Effect of raloxifene on cardiovascular events and breast cancer in postmenopausal women. N Engl J Med, 355:125-37. 
Bischoff-Ferrari HA, Willet WC, Wong JB, et al. 2005. Fracture prevention with vitamin D supplementation. JAMA, 293:2257-64.

Black DM, Cummings SR, Karpf DB, et al. 1996. Randomised trial of effect of alendronate on risk of fracture in women with existing vertebral fractures. Lancet, 348:1535-41.

Chandler JM, Zimmerman SI, Girman CJ, et al. 2000. Low bone mineral density and risk of fracture in whiter female nursing home residents. JAMA, 284:972-7.

Cummings FJ, Gray R, Tormey DC, et al. 1993. Adjuvant tamoxifen versus placebo in elderly women with node-positive breast cancer: long term follow-up and causes of death. J Clin Oncol, 11:29-35.

Cummings SR, Eckert S, Krueger KA, et al. 1999. The effects of raloxifene on risk of breast cancer in postmenopausal women. Results from the MORE (Multiple Outcomes of Raloxifene Evaluation) randomized trial. JAMA, 281:2189-97.

Deal C, Omizo M, Schwartz EN, et al. 2005. Combination teriparatide and raloxifene therapy for postmenopausal osteoporosis: Results from a 6-mont double-blind placebo-controlled trial. J Bone Miner Res, 20:1905-11.

Delmas PD, Bjarnason NH, Mitlak BH, et al. 1997. Effects of raloxifene on bone mineral density, serum cholesterol concentration, and uterine endometrium in postmenopausal women. $N$ Engl J Med, 337:1641-47.

Emkey RD, Ettinger M. 2006. Improving compliance and persistence with bisphosphonate therapy for osteoporosis. Am J Med,119(4 Suppl 1): S18-24.

Ersoy EF. 2006. Osteoporosis in the elderly with kidney disease. Int Urol Nephrol, 38 .

Ettinger B, Black DM, Mitlak BH, et al. 1999. Reduction of vertebral fracture risk in postmenopausal women with osteoporosis treated with raloxifene. JAMA, 282:637-89.

Greenspan SL, Schneider DL, McClung MR, et al. 2002. Alendronate improves bone mineral density in elderly women with osteoporosis resideng in long-term care facilities. Ann Intern Med, 136:742-6.

Hansdóttir H, Franzson L, Prestwood K, et al. 2004. The effect of raloxifene on markers of bone turnover in older women living in long-term care facilities. JAGS, 52:1-5.

Hernández E Valera R, Alonzo E, et al. 2003. Effects of raloxifene on bone metabolism and serum lipids in postmenopausal women on chronic hemodialysis. Kidney Int, 63:2269-74.

Johnell O, Scheele WH, Lu Y, et al. 2002. Additive effects of raloxifene and alendronate on bone density and biochemical markers of bone remodelling in postmenopausal women with osteoporosis. J Clin Endocrinol Metab, 87:985-92.

Kannus P, Parkkari J, Niemi S, et al. 2000. Prevention of hip fracture in elderly people with use of a hip protector. $N$ Engl J Med, 343:1506-13.

Lawrence Riggs B, Hartmann LC. 2003. Selective oestrogen-receptor modulators - mechanism of action and application to clinical practise. N Engl J Med, 347:618-29.

Liberman UA, Weiss SR, Brölll J, et al. 1995. Effect of oral alendronate on bone mineral density and the incidence of fractures in posmenopausal osteoporosis. N Engl J Med, 333:1437-43.
McClung MR, Geusens P, Miller PD, et al. 2001. Effect of risedronate on the risk of hip fracture in elderly women. $N$ Engl J Med, 344:333-40.

McPherson K, Steel CM, Dixon JM. 2000. ABC of breast diseases. Breast cancer epidemiology, risk factors and genetics. BMJ, 321:624-8.

Neer RM, Arnaud CD, Zanchetta JR, et al. 2001. Effect of parathyroid hormone on vertebral bone mass and fracture incidence among postmenopausal women with osteoporosis. N Engl J Med, 344:1434-41.

Prestwood KM, Guiness M, Muchmore D, et al. 2000. A comparative effect of raloxifene and estrogen on bone in postmenopausal women. $J$ Clin Endocrinol Metabol, 85:2197-202.

Reginster JY, Seeman E, De Vernejoul MC, et al. 2005. Strontium ranelate reduces the risk of non-vertebral fractures in women with postmenopausal osteoporosis. J Clin Endocrinol Metab, 90:2816-22.

Reid IR, Brown JP, Burckhardt P, et al. 2002. Intravenous Zoledronic acid in postmenopausal women with low bone mineral density. $N$ Engl J Med, 346:653-60.

Riggs BL. 2000. The mechanism of estrogen regulation of bone resorption. $J$ Clin Invest, 106:1203-4.

Riggs BL, Melton LJ II. 2002. Bone turnover matters: the raloxifene treatment paradox of dramatic decreases in vertebral fractures without commensurate increase in bone density. J Bone Miner Res, 17:11-14.

Sarkar S, Mitlak BH, Wong M, et al. 2002. Relationships between bone mineral density and incident verebral fracture risk with raloxifene therapy. J Bone Miner Res, 17:1-10.

Silverman SL, Minshall ME, Shen W, et al. 2001. The relationship of health-related quality of life to prevalent and incident vertebral fractures in postmenopausal women with osteoporosis. Arthritis Rheum, 44:2611-19.

Steingrimsdóttir, L, Gunnarsson Ö, Indriðason ÓS, et al. 2005. Relationship between serum parathyroid hormone levels, vitamin D sufficiency and calcium intake. JAMA, 294:2336-41.

Stevenson M, Lloyd M, De Nigris E, et al. 2005. A systematic review and economic evaluation of alendronate, etidroneate, risedronate, raloxifene and teriparatide ofr the prevention and treatment of postmenopausal osteoporosis. Health Technology Assessment, 9:1-160.

Villareal DT, Binder EF, Williams DB, et al. 2001. Bone mineral density responses to estrogen replacement in frail elderly women. JAMA, 286:815-20.

Vogel VG, Costantino JP, Wickerham DL, et al. 2006. Effects of tamoxifen vs raloxifene on the risk of developing invasive breast cancer and other disease outcomes. The NSABP Study of Tamoxifen and Raloxifene (STAR) P-2 Trial. JAMA, 295:E1-15.

Vogelvang TE, van der Mooren MJ, Mijatovic V, et al. 2006. Emerging selective Estrogen Receptor Modulator. Drugs, 66:191-221.

Walsh BW, Kuller LH, Wild RA, et al. 1998. Effects of raloxifene on serum lipids and coagulation factors in healthy postmenopausal women. JAMA, 279:1445-51. 